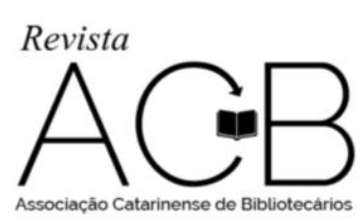

\title{
WEBOMETRIA E A ANÁLISE DE REDES SOCIAIS
}

\section{Ilaydiany Oliveira Silva ${ }^{1}$}

RESUMO: O presente artigo aborda a relação existente entre a webometria e a análise de redes sociais (ARS). Utiliza como metodologia um estudo bibliográfico através de materiais informacionais. Objetiva identificar como a webometria pode utilizar a ARS para mensurar o nível de relações existente entre os sites, buscando compreender como se dá essas relações e posições de cada site institucional dentro de uma rede social. Justifica a relevância deste estudo em compreender como se dá a comunicação cientifica entre sites institucionais. Assevera que é possível utilizar a ARS como indicador webométrico para mensurar as relações existentes entre os sites de determinadas instituições, de modo a compreender como eles se destacam no âmbito web.

Palavras-chave: Webometria. Análise de redes sociais. Visibilidade. Comunicação científica.

\section{INTRODUÇÃO}

A webometria é um método de estudo quantitativo e qualitativo das informações disponíveis na web através dos links dos sites. Este método mensura a presença de determinados sites na rede, identificando sua visibilidade, sua luminosidade e a relação existente entre sites de um mesmo universo de pesquisa, podendo assim identificar quais possuem uma boa disponibilidade de suas informações no âmbito virtual de modo a identificar as instituições que se destacam quanto as suas relações sociais na web.

A análise de redes sociais tem por objetivo estudar a estrutura social baseada no comportamento dos indivíduos de modo a identificar as relações e interações existentes entre eles, buscando demonstrar as estruturas que ocorrem em uma rede social.

Nesta perspectiva, este artigo apresenta como tema de estudo a relação entre o método webométrico e a ARS, buscando realizar uma revisão de literatura para identificar quais

\footnotetext{
1 Doutoranda em Ciência da Informação pela UFRJ/IBICT. Mestra em Engenharia de Produção pela UFRN, na área de métodos e modelos de pesquisa operacional (2011) e graduação em Biblioteconomia pela Universidade Federal do Rio Grande do Norte (2008). Participa do Grupo de Pesquisa em Estudos Métricos da Informação em Ciência, Tecnologia e Inovação (EMICT\&I) do Departamento de Ciência da Informação da Universidade Federal do Rio Grande do Norte. E-mail: ilaydiany18@ hotmail.com
} 


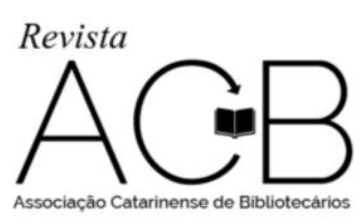

conceitos sobre redes sociais são utilizados em trabalhos voltados para os estudos métricos da informação e como a ARS pode contribuir com a webometria no tocante a identificar as relações existentes entre os sites analisados por este método.

O objetivo do estudo é identificar como a webometria pode utilizar a ARS para mensurar o nível de relação existente entre os sites, buscando compreender como se dá as relações e posições de cada site institucional dentro de uma rede social. Assim será possível compreender os fatores que favorecem a visibilidade e comunicação científica de determinados sites no âmbito web.

Para a realização deste estudo foi feita inicialmente uma incursão pela literatura buscando identificar artigos, livros, teses e dissertações que abordam a temática da webometria e da ARS, com o propósito de compreender mais profundamente as temáticas e fazer um levantamento teórico-conceitual dos dois métodos apresentados. Posteriormente, foram analisados os trabalhos que aplicam o método de ARS dentro do estudo webométrico com o pressuposto de verificar a metodologia empregada para a realização do método e sua exequibilidade.

Este trabalho deu-se a partir da inquietação da necessidade de uma maior divulgação do método de ARS associado aos estudos métricos informacionais, buscando dessa forma contribuir no processo de representação do fluxo informacional da comunicação científica de sites institucionais.

\section{WEBOMETRIA: UMA PERSPECTIVA TEÓRICO-CONCEITUAL}

$\mathrm{Na}$ atualidade há uma disseminação muito rápida das informações e este fato dar-se pelo advento das Tecnologias de Informação e Comunicação (TICs) e, notadamente, pela web que dispõe de recursos tecnológicos capazes de difundir as informações em tempo até então inimagináveis, trazendo assim diversas mudanças ao contexto social, político, econômico, entre outros.

No tocante as informações científicas, observa-se que este fato também trouxe mudanças relevantes ao meio acadêmico e científico. Pois, através das TICs é possível transferir as informações científicas de forma rápida e ágil, de modo a contribuir primordialmente com o desenvolvimento de pesquisas e estudos capazes de resolver ou minimizar diversos problemas existentes que afetam a sociedade. 


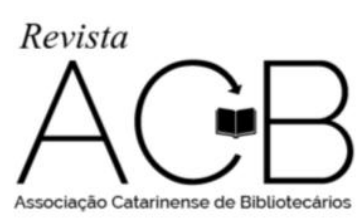

O excesso de informações disponibilizadas também proporciona uma desvantagem, cuja é identificada pela dificuldade de selecionar as informações consideradas pertinentes em meio àquelas que podem ser consideradas lixo informacional.

Nesse mesmo cenário, Lang, Gouveia e Leta (2008, p. 137) despertam a atenção de outro fator relevante quando afirmam que:

As novas tecnologias da informação e, sobretudo, a Internet, passam a contribuir para a formação de um novo modelo comunicacional, em que emissor e receptor desempenham papéis ainda mais complexos. Não Basta que as instituições estejam na Internet; deve-se refletir sobre como elas desejam ser percebidas e de que maneira estão se relacionando. (LANG; GOUVEIA; LETA, 2008, p.137).

Diante dessa dificuldade referenciada pelos autores acima, se faz necessária à inserção de métodos quantitativos que objetivam mensurar as informações científicas relevantes disponibilizadas pelas mídias informacionais, bem como identificar as relações sociais que proporcionam a comunicação científica. Dentre os estudos métricos existentes, ressalta-se a presença do estudo webométrico que tem a finalidade de quantificar e qualificar as informações disponibilizadas no âmbito virtual e assim identificar a qualidade das informações que estão sendo difundidas.

Diante da relevância desse método, será abordado a seguir um contexto conceitual do método webométrico de modo a contribuir com a compreensão sobre o tema.

\subsection{A WEBOMETRIA NO CONTEXTO CIENTÍFICO}

A webometria é um método quantitativo e qualitativo capaz de medir as informações científicas disponibilizadas na web através do uso de links. Ao mensurar as informações é possível estabelecer a relação social existente entre os sites de determinadas instituições e verificar como tem se dado a comunicação científica entre elas.

Para Daniel e Facca (2010) a webometria é tida como uma disciplina científica que estuda aspectos quantitativos e os recursos de informação em documentos web e sua utilização é baseada na estrutura de links disponibilizados na web e nos motores de busca.

Já para Björneborn (2004, p. 34) a webometria é o "estudo dos aspectos quantitativos da construção e uso dos recursos de informação, estruturas e tecnologias na web, utilizando enfoques bibliométricos e informétricos". 


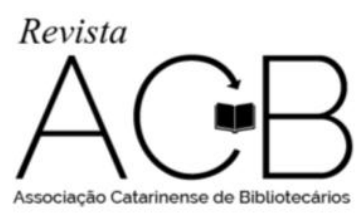

O termo webometria foi cunhado inicialmente por Almind e Ingwersen em 1997 como sendo "webometrics". No Brasil é utilizado o vocábulo webometria pelo fato de ser uma derivação da bibliometria e da informetria, métodos já trabalhados pela Ciência da Informação.

A webometria utiliza-se de ferramentas e indicadores capazes de medir a presença de determinados sites na rede, bem como sua relevância no mundo virtual e apontar quais destes sites oferecem um maior índice de perspectiva de contribuição para a ciência ao utilizar a web como suporte para a comunicação científica na atualidade. (SILVA, 2011).

Através desses recursos pode-se também avaliar a evolução da presença de uma determinada instituição ou país na rede, assim como também é possível quantificar o crescimento ou perda de importância relativa de um tema ou área do conhecimento na rede web. (VANTI, 2007).

Nessa perspectiva, observa-se a relevância de aplicar o método webométrico em sites de instituições acadêmicas de modo a verificar como está sendo a comunicação científica entres estas, bem como identificar suas relações e fatores que favorecem seu maior reconhecimento no âmbito virtual.

\section{COMPRENDENDO A ANÁLISE DE REDES SOCIAIS}

A rede social é um termo bastante presente nos dias atuais, principalmente para simbolizar as relações entre pessoas e grupos no âmbito web. Porém o termo rede social é uma derivação do vocábulo "rede" que é associado ao adjetivo "social" com o objetivo de especificar o campo das Ciências Sociais. Assim, as redes sociais podem ser compreendidas como um estudo que permite a construção de uma nova sociedade, na qual a relação social se estabelece em função dos papéis estabelecidos pela sociedade. (MARTELETO, 2010).

Nessa mesma perspectiva, as redes sociais podem ser consideradas como um conjunto de pessoas, instituições, ou organizações que, por possuírem afinidades em comum, compartilham informações e por meio dessas ligações, vão construindo e reconstruindo uma estrutura social. (TOMAÉL; MARTELETO, 2006).

A partir dos conceitos de rede social apresentados acima é possível depreender que a ARS é diferente das análises sociais que estudam a conduta dos atores sociais, pois a ARS estuda as relações entre os atores no tocante as suas características, através de indicadores de 


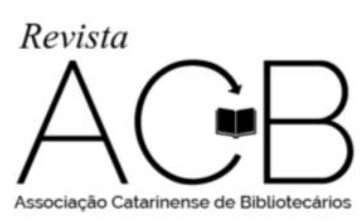

centralidade, densidade, proximidade e intermediação, conforme apresentadas no estudo Alcântara (2014) e relacionadas abaixo:

- Grau de centralidade: representa o número de atores que um ator está diretamente ligado;

- Densidade: é o quociente entre o número de relações existentes e as relações possíveis, considerando todos os atores que a formam;

- Grau de proximidade: representa a capacidade de um nó se ligar a todos os atores de uma rede;

- Grau de intermediação: é a possibilidade que um ator tem para intermediar as comunicações entre pares de nós.

Assim, é perceptível que a ARS estuda minuciosamente as relações existentes em um determinado grupo social, buscando identificar características intrínsecas que forma cada ligação através dos atores envolvidos nestas relações.

Nessa perspectiva, é relevante ressaltar a relação dos conceitos de informação, rede e rede social, buscando explanar que a informação é o elemento norteador para a formação de uma rede, visto que este elemento ao ser difundido entre os indivíduos constitui assim uma rede. A rede social pode ser assim caracterizada pela relação entre indivíduos que possuem interesses informacionais em comum.

Assim, as pessoas tendem a pertencer a uma ou mais rede, visto que estas são consideradas veículos de poder e influência das organizações e com o advento da Internet a importância de participação em redes sociais ganhou mais atenção entre os indivíduos, e, consequentemente, entre os pesquisadores sociais.

Segundo Haythornthwaite (2009) Quando há uma relação entre pessoas há a transferência de informações através da contação de histórias, discussão de ideias, partilhamento de opiniões e outras experiências, fazendo assim uma disseminação de conhecimentos entre um ou mais indivíduos.

Quando ocorre a transferência da informação se constitui assim uma conexão com base em uma decisão sobre quais informações revelar, onde, quando e para quem. Reunidos, os laços formados por transferência de informações entre os atores constroem redes com rotas ao longo do qual a informação costuma viajar, como também pontes que levam a informação de um círculo social para outro, onde a informação são becos sem saída ou circula repetidamente entre o mesmo grupo de amigos (HAYTHORNTHWAITE, 2009).

Tendo por base esse entendimento de informação, rede e rede social, pode-se observar que a rede social é algo que transcende séculos e conforme Marteleto (2001, p. 72) afirma "o 


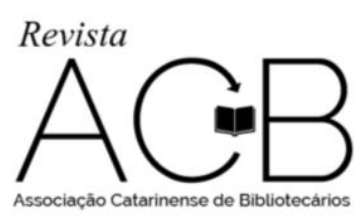

trabalho pessoal em redes de conexões é tão antigo quanto à história da humanidade, mas, apenas nas últimas décadas, as pessoas passaram a percebê-lo como uma ferramenta organizacional".

Dessa forma, se desperta o interesse em compreender as redes sociais e seu impacto ao longo do tempo, de modo a compreender como a ARS passou a ser um método de estudo para representar as relações sociais. Com este pressuposto, será detalhado a seguir um pouco mais sobre a introdução da ARS nas Ciências sociais.

\subsection{ARS: UMA PERSPECTIVA HISTÓRICA NAS CIÊNCIAS SOCIAIS}

Tendo por base o texto de Borgatti (2009) intitulado Network analysis in the social sciences é possível depreender que a Análise de redes sociais foi inicialmente estudada nas Ciências sociais em um estudo realizado por Jacob Moreno em 1932 através do método sociométrico, que trata-se de uma análise de redes com fundamentos físicos, conhecido como física social.

O estudo de Jacob Moreno foi baseado em uma análise de redes sociais de 14 jovens que haviam fugido da escola Hudson. O estudo foi importante, pois mostrou que apesar das meninas que fugiram não saberem da rede que estavam inseridas, todas participavam de uma rede interligada. Através do estudo foi percebida que a razão das fugas tinha menos a ver com fatores ligados a personalidade das meninas e sim com a localização dessas meninas na rede em que elas estavam inseridas. É relevante destacar que Jacob Moreno não foi o primeiro pesquisador a estudar sociometria, pois pesquisadores com o sociólogo Durkeim e o filósofo Comte também trabalharam nessa temática em tempos anteriores.

Borgatti (2009) afirma que nos anos de 1940 e 1950 pôde-se observar um aumento de estudos voltados para as redes sociais devido à adoção do uso da álgebra matricial, da teoria dos grafos e do desenvolvimento de um programa de laboratório de experimentação em redes.

Em 1950, Kochen - um matemático e cientista político- escreveu um artigo que ganhou uma grande repercussão apenas em 1978. O artigo escrito por Kochen tratava sobre o "mundo pequeno" abordando a problematização das possíveis ligações sociais entre dois indivíduos. Este estudo serviu de base para que sociólogos estudassem as relações concretas entre as pessoas, tais como o ódio, apoio, amor, dentre outros, de modo a representar através de uma rede a estrutura da comunidade e suas relações. 


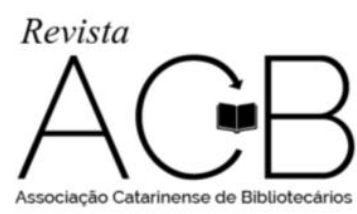

$\mathrm{Na}$ década de 60 o estudo das redes sociais ganhou uma maior repercussão na antropologia, através dos trabalhos dos pesquisadores Radcliffe Brown, Nadel, Levi-Strauss e Bott que analisavam as relações familiares e sócio afetivas como agentes de designação do desempenho das atividades e funções na cadeia social.

Em 1970 a pesquisa em rede se propagou à área da sociologia buscando definir as posições estruturais sociais como uma rede, sendo analisados os papéis e funções de cada indivíduo na rede e sua influência.

Outra contribuição importante foi a teoria desenvolvida por Granovetter, conhecida como teoria dos laços. Granovetter apresentou as estruturas de laços fortes e laços fracos, na qual ele aborda que os laços fortes tendem a serem agrupados através de um contato mais próximo entre os indivíduos da rede, já os laços fracos são existentes entre meros conhecidos, inclusive estes laços podem ser facilmente desfeitos, mas também podem trazer informações novas para a rede. (BORGATTI, 2009).

Posteriormente, os trabalhos de Granovetter desencadearam um estudo sobre a teoria geral da evolução do capital social, no qual se pressupõem que as pessoas ao estarem conectadas umas às outras possuem uma maior possibilidade de terem recursos que possibilitem melhorias em suas vidas, tais como empregos e promoções.

Na década de 80 a ARS torna-se um campo nas ciências sociais, tendo uma organização profissional, uma conferência anual, software especializado e seu próprio jornal. Já na década de 90 passa a ser difundida em diversas outras áreas, como a física e a biologia e em campos aplicados como a consultoria de gestão e a saúde pública, sendo a segurança nacional um dos campos que tem estudado mais enfaticamente, buscando minimizar problemas que envolvem o crime organizado e a formação de grupos terroristas.

De modo geral é possível verificar através do texto de Borgatti (2009) que a ARS vem sendo trabalhada desde meados de 1930 e que apesar de ter um contexto histórico fundamentado na física e na biologia, a ARS foi se difundindo em outras áreas e campos, e, notadamente, nas ciências sociais. Tendo ganhado um caráter descritivo e teórico, evidenciando sua qualidade, tipologias, estruturas e características. 


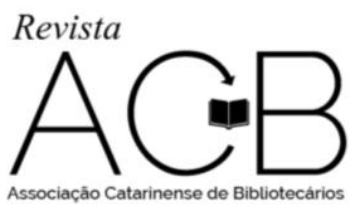

\section{A ANÁLISE DE REDES SOCIAIS APLICADA EM ESTUDOS WEBOMÉTRICOS}

Os estudos webométricos utilizam em sua aplicação ferramentas e indicadores que coletam os dados existentes nos sites e após serem analisados e interpretados contribuem na verificação da presença de determinados sites na rede.

Dentre as ferramentas utilizadas para coletar os dados webométricos, cita-se: Motores de busca, também conhecidos como sites de busca, estes têm como finalidade contabilizar o número total de páginas, documentos em determinados formatos e links em um espaço web; Programas mapeadores que são softwares que quantificam as unidades de informação; e Programas para análise e visualização de redes que representam graficamente as relações, comportamentos e propriedades estabelecidas entre diversos atores em uma rede de interação social na web, utilizando-se de softwares especializados que possibilitam avaliar os vínculos sociais.

Através destas ferramentas é possível fazer o levantamento dos links a serem utilizados na pesquisa e após a mineração desses dados é possível aplicá-los nos indicadores webométricos.

No processo de avaliação dos dados obtidos são utilizados os indicadores webométricos: tamanho dos sites; visibilidade; fator de impacto web (FIW); luminosidade; e densidade da rede (DR). E para uma melhor compreensão, os indicadores a serem utilizados serão detalhados a seguir:

I. TAMANHO DOS SITES: Define a quantidade de páginas que determinado site possui na web, seu tamanho é estabelecido através da soma de todas as páginas que fazem parte de um mesmo domínio. (VANTI, 2007).

II. VISIBILIDADE: Indica como determinado site é conectado ou "citado" pela rede através dos inlinks de domínios de outras instituições, ou seja, relaciona a quantidade de páginas de outras instituições que "linkam" para o site analisado. (WEBOMETRICS, 2010).

III. FATOR DE IMPACTO WEB (FIW): Serve para medir e comparar a atratividade de sites da web, baseando-se na soma do número de páginas que levam a um determinado site (visibilidade), dividido pelo logaritmo neperiano do número de páginas deste site (tamanho), apresentado de acordo com Vanti, Silva, Costa, (2012) sob a seguinte fórmula:

$$
F I W=\frac{\text { Visibilida de }}{\ln (\text { Tamanho })} \quad \text { IV. } \begin{aligned}
& \text { (Fórm } \\
& \text { ula 1) }
\end{aligned}
$$




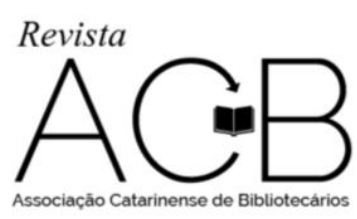

V. LUMINOSIDADE: analisa o número de links externos que um site apresenta, apontando para outros sites ou URLs, ou seja, verifica como o site analisado aponta o resto da web, indicando o seu grau de conectividade. (RODRÍGUEZ, 2006).

VI. DENSIDADE DA REDE: analisa as redes sociais na web, de forma a mostrar o quanto uma população se relaciona entre si. Para tanto, torna-se imperativo estabelecer a população que se deseja estudar ("nós”) dentro da web e, após, identificar todas as relações (links) existentes entre estes "nós". Contudo, divide-se o número de relações (links) pela quantidade de "nós" da população estudada, multiplicando por este mesmo número menos 1. Como mostra a fórmula, sugerida por Vanti (2007), a seguir:

$$
D R=\frac{\operatorname{links}}{n \cdot(n-1)}
$$

De acordo com Aguillo (2003) os indicadores webométricos podem ser divididos em quatro grandes grupos, definidos por: descritivos, de conectividade, de impacto e de densidade.

O grupo descritivo contabilizam as páginas, os links, os arquivos que são recuperados pelas ferramentas webométricas e quantifica a participação das instituições na web, no tocante a quantidade de informações disponibilizadas no meio virtual.

O grupo de conectividade, de impacto e de densidade trata da relação dos hipertextos existentes na web e tem por finalidade o exame das conexões entre páginas e sítios por meio dos seus links.

Dentre os indicadores utilizados pela webometria, destaca-se neste estudo a densidade de rede, o qual estabelece as relações existentes entre os sites estudados. Através deste indicador que são verificadas as conexões ou citações que ocorrem entre os sites.

As medidas de densidade especificamente têm como propósito estimar o quanto uma população se relaciona entre si dentro de uma rede ou comunidade virtual, tendo em vista o número máximo de possibilidades de relacionamentos. (VANTI, 2007).

A densidade da rede é um indicador também utilizado na ARS para mostrar o quanto uma população se relaciona entre si. Na webometria, este indicador é utilizado após se fazer um levantamento de todos os links da população que se pretende estudar dentro de uma rede, ou seja, os nós que fazem parte dela. Logo após, busca-se identificar todas as conexões de links (inlinks e/ou outlinks) que existem nestes nós, também chamadas de "sitações" - neologismo criado por McKiernan (1996) para designar as citações que ocorrem entre sites - 


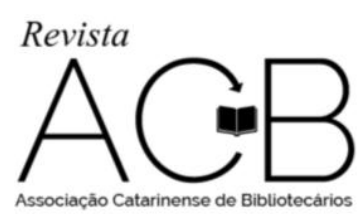

de modo a construir uma matriz de adjacência que por sua vez relacionará as relações existentes entre os nós.

Na FIGURA 1 é representada uma matriz de adjacência de 7 sites intitulados pelas letras do alfabeto, a representação mensura as "sitações" existentes entre os sites, de modo a apresentar como esses sites se comunicam e quais possuem relações sociais com os demais. Na figura é possível estabelecer quantos links há dentro de um site e que remete para os demais, quantificando assim seu grau de comunicação virtual e, consequentemente, seu nível de relação social.

Figura 1 - Matriz de adjacências

Fonte: Elaborado pela autora.

\begin{tabular}{|l|c|c|c|c|c|c|}
\hline & SITE A & SITE B & SITE C & SITE D & SITE E & SITE F \\
\hline SITE A & 0 & 5 & 0 & 1 & 0 & 2 \\
\hline SITE B & 0 & 0 & 0 & 1 & 1 & 1 \\
\hline SITE C & 2 & 3 & 0 & 0 & 0 & 0 \\
\hline SITE D & 2 & 0 & 0 & 0 & 0 & 1 \\
\hline SITE E & 1 & 0 & 1 & 0 & 0 & 1 \\
\hline SITE F & 1 & 0 & 0 & 1 & 1 & 0 \\
\hline
\end{tabular}

Após a construção da matriz de adjacências é estabelecida a relação entre os atores através da construção dos grafos de rede (FIGURA 2) e assim torna-se possível a visualização das redes sociais dos sites, sendo possível identificar os sites que possuem laços fortes e laços fracos na rede, de modo a analisar quais fatores informacionais podem favorecer o destaque de determinado sites na web. Para a construção das matrizes e dos grafos é possível utilizar softwares como o Pajek, o Ucinet6 e o Netdraw e Mage, que são os mais conhecidos e utilizados no Brasil. 
Figura 2 - Representação do grafo de rede

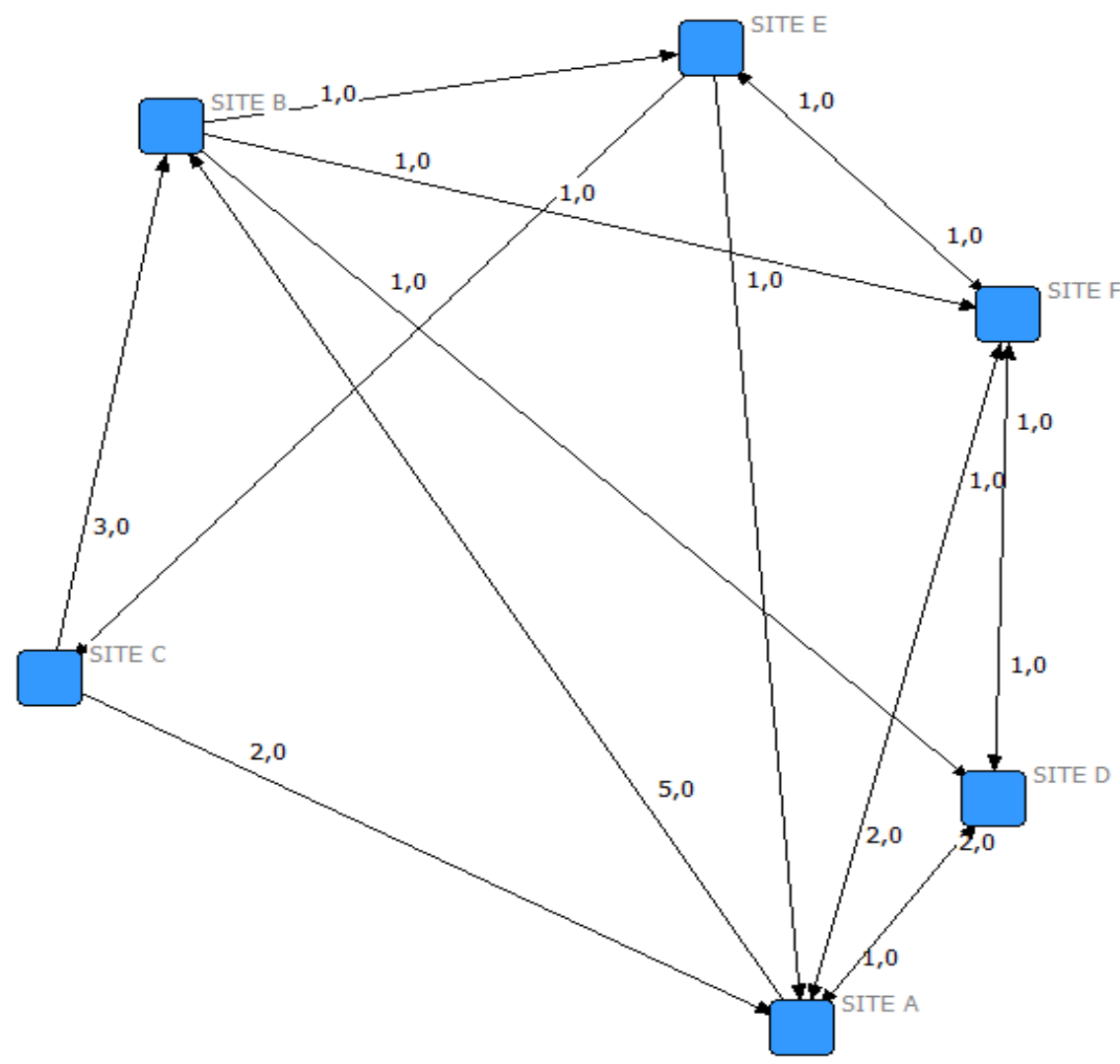

Fonte: Elaborado pela autora.

A partir da representação por grafos, diversas informações importantes podem ser extraídas, pois ao se observar o exemplo acima se pôde verificar as relações sociais simbolizadas por suas setas e nós, de modo que os sites A e F são aqueles que recebem mais "sitações" vindas de outros sites, em contrapartida o site A disponibiliza em suas páginas 5 links para o site $\mathrm{B}$, fato que demonstra o intenso fluxo informacional que ocorre nessa rede social estabelecida. O site $\mathrm{C}$ é aquele que recebe a menor quantidade de "sitações" para sua página, em contrapartida disponibiliza números significantes de links que remetem para os sites B e A. Ao se fazer uma análise geral dos atores da rede é possível inferir que o site A é aquele que possui laços mais fortes, destacando-se como o ator principal da rede, pois apresenta um número considerável de relações sociais.

Diante da relevância de se aplicar a ARS na webometria, Alcântara (2014, p. 20) detalha a relevância dos grafos para representar as relações sociais em diversos âmbitos. 


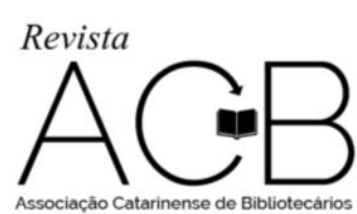

Os grafos são uma representação matemática adequada para trabalhar com redes sociais e sítios na web. Nas redes sociais, os perfis, ou páginas dos indivíduos são tratados como os nós, ou vértices do grafo, enquanto as relações podem ser tratadas como as arestas que ligam estes vértices. As ligações podem estar relacionadas a um vínculo de amizades, a um interesse em comum ou a um grupo ao qual os dois atores pertencem. Dentro do contexto de websites, os sítios podem ser tratados como os atores (nós) e os links ou ligações como sendo as arestas. (ALCÂNTARA, 2014, p. 20)

Nesse mesmo entendimento, Vanti (2007, p. 79) ressalta a relevância da visualização das redes sociais através dos grafos, quando afirma que:

A visualização das redes sociais e a análise de suas propriedades por meio de softwares associados é um dos campos mais promissores da 'Análise de Redes Sociais' (ARS) por incorporar nos desenhos conceituais oferecidos por tais softwares o princípio sociológico, tendo como fundamento o valor dos vínculos e guardando, sobretudo, uma relação muito estreita com a teoria dos grafos do campo da matemática. Considerada como base para o desenvolvimento da ARS, esta teoria possibilita estudar o comportamento das redes e conhecer suas propriedades.

Tendo por base as asseverações acima é notório que a ARS quando aplicada em estudos webométricos contribui diretamente na visualização e compreensão das relações entre os sites, favorecendo o entendimento das comunicações existentes entre as instituições que são representadas através das URLs dos sites analisados.

A ARS relaciona-se ao estudo das interações entre uma série definida de nós ou atores (os websites) e os laços que interligam estes nós (links), viabilizando a construção de matriz relacional e representação gráfica, além do cálculo de medidas de estrutura e posição (ZAMBALDE et al, 2011).

De modo geral, observa-se que o método webométrico quando associado a ARS pode trazer resultados bastante pertinentes para a compreensão das relações existentes entre os sites das instituições, podendo representar graficamente os laços fortes e fracos existentes entre as instituições no âmbito virtual. Consequentemente, é possível identificar quais fatores informacionais possibilitam a citação entre estes sites e assim como é formada as relações entre os sites analisados no estudo webométrico.

\section{CONCLUSÕES}

A partir do estudo aqui proposto foi possível fazer uma incursão pela literatura sobre a relevância de aplicar a ARS e como a mesma possui relações pautadas nos objetivos dos 


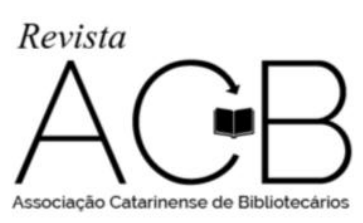

indicadores webométricos. Nesse contexto, foi possível verificar a eficiência nas análises sobre a comunicação científica existente no âmbito virtual.

O estudo aqui proposto atingiu seu objetivo, pois foi capaz de identificar através da aplicação da ARS quais os laços fortes e fracos existentes entre as instituições que dispõe suas informações na web, como forma de identificar quais são os sites que apresentam um destaque quanto suas "sitações" através de seus links e como estas podem contribuir com a identificação de atores principais que alimentam a transferência e a comunicação de informações na web.

Quando analisado os materiais bibliográficos utilizados para a elaboração deste artigo foi possível depreender que no Brasil os estudos webométricos que aplicam a ARS ainda são relativamente poucos, mas dentre estes os resultados apresentados em suas pesquisas proporcionam uma maior análise qualitativa das relações existentes entre os sites analisados e assim é torna-se mais fácil identificar como é o fluxo da comunicação cientifica destes sites.

Contudo, foi percebido que utilizar a ARS como indicador webométrico para representar as relações existentes entre os sites de determinadas instituições, contribui diretamente no entendimento dos fatores informacionais que favorecem a citação entre estes sites e que as relações quantificadas nos indicadores de visibilidade e luminosidade podem ser mais bem interpretadas ao se estabelecer uma representação gráfica das redes sociais dos sites.

Por fim, corrobora-se que quando aplicado o método da ARS nos estudos webométricos há uma maior representatividade dos aspectos qualitativos da construção e uso dos recursos de informação, estruturas e tecnologias da web na análise dos sites.

\section{REFERÊNCIAS}

AGUILLO, Isidro F. Cibermetría: la métrica de la Web. In: SEMINÁRIO BUSQUEDA: DEL ARCHIVO A LA RED. Madrid: Residencia de Estudiantes Fundación Francisco Giner de los Ríos, 2003.

ALCÂNTARA, Danielle Aparecida. Webometria e análise de rede (twitter): estudo em universidades federais no estado de Minas Gerais. 2014. 60 f. Monografia (Graduação) Curso de Ciência da Computação, Ciência da Computação, Universidade Federal de Lavras, Lavras, MG, 2014. 


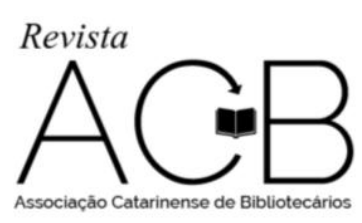

ALMIND, Tomas C.; INGWERSEN, Peter. Informetric analyses on the world wide web: methodological approaches to 'Webometrics'. Journal of Documentation, v. 53, n. 4, p. 404-426, 1997.

BJÖRNEBORN, Lennart. Small-worlld structures across an academir web space: a library and information science approach. PHD dissertation. Copenhagen, DK: Departament of Informations Studies, Royal School of Library and Information Science, 2004.

BORGATTI, Stephen P. et al. Network analysis in the social sciences. Science. v. 323, p. 892-895. 2009.

DANIEL, Florian; FACCA, Michele Federico. Current trends in web engineering. Germany: Springer, 2010.

HAYTHORNTHWAITE, Caroline. Social networks and Information transfer. In: The Enciclopedia of Library and Information Science. N.Y.: Taylor \& Francis, Márcia Bates \& Mary Maack Eds, pre-print, 2009

LANG, P. B. ; GOUVEIA, F. C. ; LETA, J. . Relações intra-institucionais na Internet: um estudo exploratório com base em metodologias webométricas. Perspectivas em Ciência da Informação, v. 13, p. 137-150, 2008.

MARTELETO, Regina. Redes sociais, mediação e apropriação de informações: situando campos, objetos e conceitos na Ciência da Informação. ANCIB, Tendências da Pesq. Bras. em C.I., v. 3, n.1, 2010.

Análise de redes sociais: aplicação nos estudos de transferência da informação.

Ciência da Informação, v.30, n.1, p. 71-81, jan. / abr., 2001. Disponível em:

<http://www.scielo.br/pdf/ci/v30n1/a09v30n1.pdf>. Acesso em 28 jul. 2015.

TOMAÉL, Ma. Inês; MARTELETO, Regina M.. Redes sociais: posições dos atores no fluxo da informação. Florianópolis: Encontros Bibli: R. Eletr. Bibliotecon. Ci. Inf., n. esp., $1^{\circ}$ sem. 2006

RODRÍGUEZ, Ailín Martínez . Indicadores cibermétricos: nuevas propuestas para medir la información en el entorno digital. 14(4), jul.-ago. 2006. Disponível em:

$<$ http://bases.bireme.br/cgi-bin/wxislind.exe/iah/online/>. Acesso em: 27 jul. 2015.

SILVA, Ilaydiany Cristina Oliveira da. Aplicação de indicadores webométricos nos programas de pós-graduação das engenharias recomendados pela Capes. $2011.138 \mathrm{f}$. Dissertação (Mestrado) - Curso de Engenharia de Produção, Programa de Pós-graduação em Engenharia de Produção, Universidade Federal do Rio Grande do Norte, Natal, 2011.

VANTI, Nadia Aurora. Links hipertextuais na comunicação científica: análise Webométrica dos sítios acadêmicos latino-americanos em Ciências Sociais. 2007. 299 f. Tese (Doutorado em Comunicação e Informação) - Curso de Programa de Pós-Graduação em Comunicação e Informação, Universidade Federal do Rio Grande do Sul, Porto Alegre, 2007. 


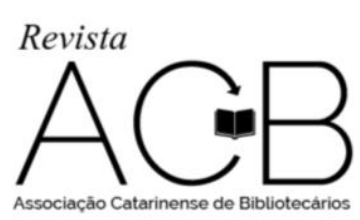

VANTI, Nadia; SILVA, I.C.O. da; COSTA, J. F. Fator de impacto web (fiw): um novo cálculo para a obtenção de resultados mais acurados. In: Anais do $3^{\circ}$ Encontro Brasileiro de Bibliometria e Cientometria. Gramado, 2012. v. 1.

WEBOMETRICS Ranking Universities. Disponível em: <http://www.webometrics.info /about_rank.html>. Acesso em: 28 jul. 2015.

ZAMBALDE, A.L. et all. Redes sociais da internet no agronegócio brasileiro: conhecimento, webometria e interação. Anais... $6^{\text {a }}$ Conferência Ibérica de Sistemas e Tecnologias de Informação, Chaves, 2011.

\section{WEBOMETRICS AND SOCIAL NETWORKS ANALYSIS}

ABSTRACT: This article explores the relationship between webometrics and social network analysis (SNA). A bibliographical study through informational materials is performed. The objective is to identify how webometrics can utilize SNA to measure the degree of relationship between institutional websites, describing those relationships and each site's position within a social network. The relevance of this study lies on the comprehension of the scientific communication between institutional websites. It is possible to utilize SNA as a webometrics indicator to measure the existing relationships between certain institutional websites, leading to an understanding of how visible they are within the web.

Keywords: Webometrics. Social network analysis. Visibility. Scientific communication. 